\title{
DISTRIBUIÇÃO ESPAÇO-TEMPORAL DA MORTALIDADE POR MALFORMAÇÕES CONGÊNITAS E CAUSAS MAL DEFINIDAS, EM ESTADOS DA REGIÃO CENTRO-SUL DO BRASIL
}

\section{SPACE AND TIME DISTRIBUTION OF MALFORMATION AND UNCERTAIN CAUSES OF DEATH IN THE BRAZILIAN STATES OF THE CENTER-SOUTH REGION}

\begin{abstract}
SôNIA CORINA HESS
Engenheira Química. Doutora em Química Orgânica pela Universidade Federal de Santa Catarina. Professora da Universidade Federal de Mato Grosso do Sul, Centro de Ciências Exatas e Tecnologia, Departamento de

Hidráulica e Transportes
\end{abstract}

OrLando Pissuto TREVISAN

Engenheiro ambiental (UFMS). Mestre em Tecnologias Ambientais (UFMS)

Recebido: 20/09/07 Aceito: 21/10/08

\section{RESUMO}

A investigação da distribuição espacial da mortalidade por malformaçōes congênitas pode ser útil em programas de vigilância ambiental em saúde. Foram analisados os padróes espaço-temporais da mortalidade por malformaçōes congênitas e causas mal definidas em Estados brasileiros (MS, MT, GO, MG, SP, PR, SC e RS) e no Distrito Federal, no período de 2000 a 2004. Os valores observados foram obtidos do Sistema de Informaçôes de Mortalidade (SIM) do Ministério da Saúde. Foi utilizado um modelo bayesiano que rende interação espaço-temporal. Muitas microrregiōes com baixas taxas para malformações congênitas tiveram valores elevados para causas mal definidas, sendo que os óbitos por causas mal definidas prejudicam a identificação de microrregiōes com taxas de mortalidade por malformaçôes congênitas acima do esperado, indicativas da existência de fatores ambientais de risco para estas doenças.

PALAVRAS-CHAVE: Malformações congênitas, mortalidade, sistema de informação, métodos bayesianos, análise espacial, análise temporal.

\section{INTRODUÇÃO}

Em média, entre 3 e $5 \%$ das crianças nascem com malformações congênitas, e as causas destes problemas, freqüentemente, não são identificadas, sendo que a fração atribuída a causas genéticas é de 20 a $25 \%$ destes casos. Presume-se que as malformaçōes congênitas sejam decorrentes de interações complexas entre a predisposição genética e fatores ambientais aos quais o feto tenha sido exposto, denominados herança multifatorial (Yaakov et al, 2006).

Há muitas evidências científicas de que diversas anomalias congênitas em animais de laboratório e em seres humanos ocorrem devido à exposição a algumas classes de poluentes presentes no ambiente, como: inseticidas - dieldrin, quepone (clordecone), endosulfan, metoxiclor, toxafeno e diazinon; herbicidas - linuron, alaclor e atrazina; fungicidas - vinclozolin, procymidona e procloraz (Sonnenschein \& Soto, 1998; Skakkebaek, Rajpert-De \& Main, 2001); metais pesados (incluindo mercúrio, cádmio, chumbo, entre outros) (Wibberley et al, 1977; Harada, 1978; Ragan \& Mast, 1990); solventes orgânicos (Olshan et al, 1991; Cordier et al, 1992; Shaw, 1992); substâncias químicas semelhantes a dioxinas, incluindo bifenilas policloradas (PCBs) (Khoury, 1989; Jacobson et al, 1990; Sonnenschein \& Soto, 1998; Skakkebaek, Rajpert-De \& Main, 2001); componentes de filtros solares, como 4-metil benzilideno cânfora (4-MBC) e 3-benzilideno cânfora (3-BC) (Schlumpf et al, 2001 e 2004; Heneweer et al, 2005; Maerkel et al, 2005; Soto \& Sonnenschein, 2005); componentes de plásticos e de outros materiais de amplo emprego, como bisfenol A (Judy et al, 1999; Markey et al, 2005; Vom Saal et al, 2005; Durando et al, 2007), ftalatos (Foster et al, 2001; Foster, 2006; 
Latini et al, 2003; Swan et al, 2005; Gray Jr. et al, 2006; Lottrup et al, 2006), alquilfenóis (Sonnenschein \& Soto, 1998; Loyo-Rosales et al, 2004); promotores de crescimento utilizados na criação de gado, como o dietilstilbestrol (DES) (Sharpe et al, 1998; Cardoso et al, 1999; Swan, 2000; Palmer et al, 2006) e o acetato de trembolona (Gray et al, 2006).

Tendo em vista os estudos que correlacionam a presença de substâncias tóxicas no ambiente, com o surgimento de malformações congênitas, a investigação da distribuição espacial da mortalidade por estes males poderia servir como instrumento a ser utilizado em programas de vigilância ambiental em saúde. Assim sendo, o objetivo do estudo foi investigar a distribuição espaço-temporal das taxas relativas de mortalidade por "malformaçôes congênitas, deformidades e anomalias cromossômicas" e por "sinais, sintomas e achados anormais em exames clínicos e laboratoriais" (causas mal definidas), nas microrregióes dos Estados de Mato Grosso do Sul, Mato Grosso, Goiás, Distrito Federal, Minas Gerais, São Paulo, Paraná, Santa Catarina e Rio Grande do Sul, no período de 2000 a 2004, a partir dos dados descritos no Sistema de Informaçóes de Mortalidade (SIM) do Ministério da Saúde (Ministério da Saúde, 2007).

No estudo foram aplicadas técnicas bayesianas, que são bastante úteis para a detecção de padrões espaciais e temporais de mortalidade por malformações congênitas e outras doenças. Em geral, os resultados das análises bayesianas são apresentados em mapas construídos a partir de taxas ajustadas, obtidas a partir do tratamento matemático da taxa de cada área e das taxas nas áreas vizinhas, modelando-se os riscos relativos de mortalidade para cada área, levando-se em consideração as informaçôes da vizinhança (Clayton \& Kaldor, 1987; Castro, Vieira \& Assunção, 2004; Santos, Rodrigues \& Lopes, 2005).

\section{METODOLOGIA}

\section{Dados}

Os valores para a mortalidade foram obtidos do Sistema de Informações de Mortalidade (SIM) do Ministério da Saúde (Ministério da Saúde, 2007) e são relativos a óbitos por "malformaçôes congênitas, deformidades e anomalias cromossômicas" (Capítulo CID-10, XVII) ou por "sinais, sintomas e achados anormais em exames clínicos e laboratoriais" (Capítulo CID-10, XVIII), segundo local de residência do indivíduo, no período de 2000 a 2004.

A exemplo do que foi adotado por Castro e colaboradores (2004), para evitar-se problemas nas taxas em pequenos municípios, já que os eventos analisados são raros, optou-se por avaliar as taxas de mortalidade relativas às microrregióes, que são grupos de municípios definidos pelo IBGE. Os valores esperados foram calculados aplicando-se a técnica de padronização indireta segundo sexo e a faixa etária menor que 01 ano; adotando-se como referências as taxas específicas por sexo e faixa etária da população de toda a regiāo sob análise. As taxas de incidência foram calculadas empregando-se o método dos estimadores bayesianos empíricos, de modo semelhante ao descrito por Santos e colaboradores (2005), utilizando-se o software TerraViewPlus 3.03 (INPE, 2007).

\section{Análise estatística}

Para o cálculo do risco de óbito por "malformaçōes congênitas, deformidades e anomalias cromossômicas" ou por "sinais, sintomas e achados anormais em exames clínicos e laboratoriais" para cada microrregião, $\xi_{\mathrm{i}}(\mathrm{i}=1 \mathrm{a} 275$ ), aplicou-se o estimador bayesiano empírico local (Castro, Vieira \& Assunção, 2004; Santos, Rodrigues \& Lopes, 2005), do software TerraViewPlus 3.03 (INPE, 2007) em que, para cada ano, sexo e faixa etária, nos campos descritos como casos foram acrescentados os dados do SIM referentes aos óbitos por uma destas causas e, nos campos relativos á população, o número total de óbitos para a faixa etária e sexo, em cada uma das 275 microrregióes dos Estados de MS, MT, GO, DF, MG, SP, PR, SC, e RS. A partir dos valores de $\xi_{\mathrm{i}}$ foram calculadas as Razóes de Mortalidade Padronizadas (Standardized Mortality Ratio - SMR, em inglês) ajustadas pelo modelo bayesiano (SMR ) para cada microrregião (i), sendo que:

$\mathrm{SMR}_{\mathrm{i}}=\left(\mathrm{p}_{\mathrm{i}} / \mathrm{E}\right)$

onde $\mathrm{E}=\mathrm{OT}_{\mathrm{c}} / \mathrm{OT}$

sendo $\mathrm{OT}_{\mathrm{C}}$ o número total de óbitos por "malformaçōes congênitas, deformidades e anomalias cromossômicas" ou por "sinais, sintomas e achados anormais em exames clínicos e laborato- riais", para cada sexo e ano, na região; e OT o número total de óbitos, para cada sexo, ano e faixa etária, na região.

A exemplo do que foi descrito por Castro e colaboradores (2004), para avaliar-se a evolução das taxas de SMR de uma mesma área ao longo dos anos, foram modelados por regressão linear os logaritmos das $S M R_{i}, \theta_{i R}$, aos invés dos riscos $S M R_{i}$ diretamente, onde:

$\mathrm{i}_{\mathrm{iR}}=\ln \left(\mathrm{SMR}_{\mathrm{i}}\right)$

$\mathrm{O}$ modelo aplicado permitiu a avaliação do crescimento das taxas SMR em cinco anos e a sua projeção para o ano de 2002, representando o valor esperado a posteriori.

\section{RESULTADOS E DISCUSSÃO}

Os valores esperados a posteriori para o ano de 2002, das razóes de mortalidade padronizada ajustadas pelo modelo bayesiano (SMR) de cada uma das 275 microrregiões avaliadas, para os óbitos por "malformações congênitas, deformidades e anomalias cromossômicas" ou por "sinais, sintomas e achados anormais em exames clínicos e laboratoriais" (causas mal definidas), faixa etária menor de 01 ano, para os sexos feminino e masculino, são apresentadas na Figura 01. Os mapas foram elaborados com os valores multiplicados por 100 , e indicam a probabilidade do $\mathrm{SMR}_{\mathrm{i}}$ para cada uma das 275 microrregiōes investigadas ultrapassar, ou não, o valor esperado para toda a região de abrangência do estudo. As regiôes com SMRi maior do que 150 (em preto nos mapas) tiveram estas taxas, no mínimo, $50 \%$ acima do valor estatisticamente projetado indicando que, naqueles locais, a incidência de mortes pelas causas investigadas está muito acima do que seria esperado.

Nos mapas referentes à mortalidade por causas mal definidas, os SMRi para os sexos masculino e feminino não apresentaram diferenças notáveis. Por outro lado, nos mapas correspondentes à mortalidade por malformaçōes congênitas, para o sexo masculino, as taxas mais altas foram observadas em microrregióes de todos os estados, exceto MG, enquanto que, para o sexo feminino, as microrregióes do estado do RS apresentaram apenas taxas baixas, enquanto que as de MG também apresentaram taxas elevadas, como nos demais estados. Muitas microrregiōes com baixo SMRi para os 
óbitos por malformações congênitas apresentaram valores elevados para os óbitos por causas mal definidas. Para as causas mal definidas, as taxas mais elevadas ocorreram, principalmente, em microrregióes dos estados de $\mathrm{MG}$, GO, SP, PR e SC.

Para uma mesma faixa etária, sexo e localização, nos anos de 2000 a 2004, a proporção de óbitos do SIM (Ministério da Saúde, 2007) atribuídos a "Sinais, Sintomas e Achados Anormais em Exames Clínicos e Laboratoriais" é relevante quando comparada aos óbitos por diversas enfermidades. Tais registros de óbito correspondem às causas de óbito mal definidas (Aidar, 2000) e prejudicam a detecção de microrregiōes com taxas de mortalidade acima do esperado, onde poderiam ser identificados fatores de risco para a saúde, presentes no ambiente. Tais problemas do SIM levaram Cavalini e Leon (2007) a elaborar uma metodologia em que foram aplicados estimadores bayesianos empíricos de James-Stein modificados, para corrigir os sub-registros de óbitos por causas mal definidas.

\section{CONCLUSÃO}

Os registros de óbito descritos como "Sinais, Sintomas e Achados Anormais em Exames Clínicos e Laboratoriais", correspondentes às causas de óbito mal definidas, prejudicam a identificação de microrregiōes com taxas de mortalidade por malformaçôes congênitas e outros problemas, acima do esperado. Portanto, constituem um importante viés na utilização dos dados do SIM em estudos visando o mapeamento de fatores de risco para doenças, presentes no ambiente.

\section{AGRADECIMENTOS}

Agradecemos especialmente a Maria Lúcia Ivo (UFMS), Sônia Pessoa (UCDB), Carmencita Lang, Marcelo Souza, Silvana Dorneles, Hilda Freitas (SES/Mato Grosso do Sul), Leonardo Rigo, Michael Honner (SMS/Campo Grande), Sérgio Koifmann (FIOCRUZ) e Maria do SocorroOliveira (INCA), pelas importantes sugestões, e a Ana Maria Rosa, Alessandra Fonseca, Karina Souza e KatrinyMichelle pela colaboração no levantamento dos dados. Este trabalho foi parcialmente financiado pela FUNDECT-MS (protocolo 41/100.129/2005).
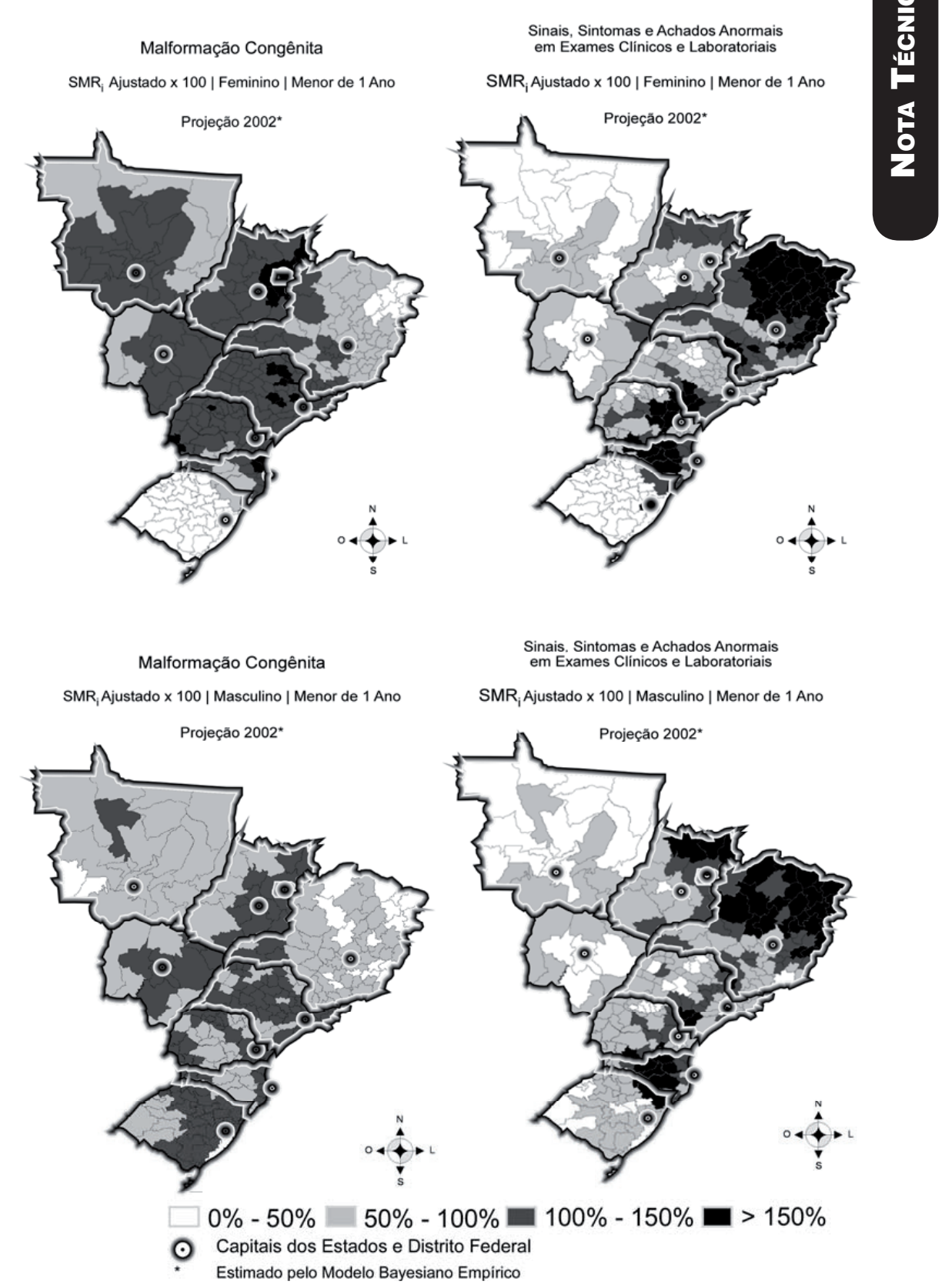

Figura I - Razões de mortalidade padronizadas (SMR) ajustadas pelo modelo, valores esperados a posteriori para o ano de 2002, região centro-sul do Brasil

\section{REFERÊNCIAS}

AIDAR, T. Avaliação da qualidade das informaçôes de causas de morte: banco de dados SIM. In: BERQUÓ, E., CUNHA, E. M. G. P., eds. Morbimortalidade Feminina no Brasil (1979-1995). UNICAMP, p. 20-38. Campinas, 2000.

CARDOSO, O. M. C. et al. Ocorrência de residuos de dietilestilbestrol e zeranol em fígado de bovinos abatidos no Brasil. Cienc. Tecnol.Alim. v. 19 , p. 305-310, 1999.

CASTRO, M. S. M., VIEIRA, V. A., ASSUNÇÃO, R. M. Padröes espaço-temporais da mortalidade por câncer de pulmão no sul do Brasil. Rev. Bras. Epidemiol. v 7, p. 131-143, 2004.
CAVALINI, L. T., LEON, A. C. M. P. Correção de sub-registros de óbitos e proporção de internaçōes por causas mal definidas. Rev Saúde Pública, v. 41, p. 85-93, 2007.

CLAYTON, D. E., KALDOR, J. Empirical Bayes estimates of age-standardized relative risks for use in disease mapping. Biometrics v. 43, p. 671-681, 1987.

CORDIER, S. et al. Maternal occupational exposure and congenital malformations. Scand. J. Work, Environment and Health. v. 18, p. 11-17, 1992.

DURANDO, M. et al. Prenatal bisphenol A exposure induces preneoplastic lesions in the mammary gland in wistar rats. Environ. Health Persp. v. 115, p. 80-86, 2007. 
FOSTER, P. M. D. et al. Effects of phthalate esters on the developing reproductive tract of male rats. Hum. Reprod. Update. v. 7, p. 231-235, 2001.

FOSTER, P. M. D. Disruption of reproductive development in male rat offspring following in utero exposure to phthalate esters. Int. J. Androl. v. 29, p. 140-147, 2006.

GRAY JR., L. E. et al. Adverse effects of environmental antiandrogens and androgens on reproductive development in mammals. Int. J. Androl. v. 29, p. 96-104, 2006.

HARADA, M. Congenital Minamata disease: intrauterine methylmercury poisoning. Teratology v. 18 , p. $285-288,1978$

HENEWEER, M. et al. Additive estrogenic effects of mixtures of frequently used UV filtres on PS2-gene transcription in MCF-cells. Toxicol. Appl. Pharmacol. v. 208, p. 170-177, 2005.

INPE - INSTITUTO NACIONAL DE PESQUISAS ESPACIAIS. Projeto TerraView. Disponível na página da internet: http://www. dpi.inpe.br/terraview/index.php Acessado em 01/09/2007.

JACOBSON, J. L. et al. Effects of exposure to PCBs and related compounds on growth and activity in children. Neurotoxicology and Teratology. v. 12, p. 319-326, 1990 .

JUDY, B. M. et al. Low-dose bioactivity of xenoestrogens in animals: fetal exposure to low doses of methoxychlor and other xenoestrogens increases adult prostate size in mice. Toxicol. Ind. Health. v. 15 , p. $12-25,1999$.

KHOURY, M. J. Epidemiology of birth defects. Epidemiologic Reviews. v. 11, p. 244-248, 1989.

LATINI, G. et al. In utero exposure to di (2-ethylhexyl) phthalate and duration of human pregnancy. Environ. Health Persp. V. 111, p. 1783-1785, 2003.

LOTTRUP, G. et al. Possible impact of phthalates on infant reproductive health. Int. J. Androl. V. 29: 172-180, 2006.

LOYO-ROSALES, J. E. et al. Migration of nonylphenol from plastic containers to water and a milk surrogate. J. Agric. Food Chem. V. 52, p. 2016-2020, 2004.
MAERKEL, K. et al. Sex- and region-specific alterations of progesterone receptor $m R N A$ levels and estrogen sensitivity in rat brain following developmental exposure to the estrogenic UV filter 4-methylbenzylidene camphor. Environ. Toxicol. Pharmacol. V. 19, p. 761-765, 2005.

MARKEY, C. M. et al. Long-term effects of fetal exposure to low doses of the xenoestrogen bisphenol $A$ in the female mouse genital tract. Biol. Reprod. V. 72, p. 1344-1351, 2005.

MINISTÉRIO DA SAÚDE. Informaçôes de saúde. Estatísticas vitais - Mortalidade e Nascidos Vivos. Disponível na página da internet: <http:// w3. datasus.gov.br/datasus/datasus.php?area $=35$ 9A1B378C5D0E0F359G22H0I1Jd5L25M0N \&VInclude=../site/infsaude.php\&VObj=http:// tabnet.datasus.gov.br/cgi/deftohtm.exe?sim/cnv/ obt> Acessado em 01/09/2007.

OLSHAN, A. F. et al. Paternal occupation and congenital anomalies in offspring. Am. J. Ind. Med. v. 20 , p. $447-475,1991$.

PALMER, J. R. et al. Prenatal diethylstilbestrol exposure and risk of breast cancer. Cancer Epidemiol. Biomarkers Prev. v. 15, p. 1509-1514, 2006.

RAGAN, H. A., MAST, T. J. Cadmium inhalation and male reproductive toxicity. Rev. Environ. Contamin. Toxicol. v. 114, p. 1-22, 1990.

SANTOS, A. E., RODRIGUES, A. L., LOPES, D. L. Aplicaçōes de estimadores bayesianos empiricos para análise espacial de taxas de mortalidade. In: VII SIMPÓSIO BRASILEIRO DE GEOINFORMÁTICA - GEOINFO, Campos do Jordão, 2005.

SCHLUMPF, M. et al. Endocrine activity and developmental toxicity of cosmetic UV filters: an update. Toxicology v. 205, p. 113-122, 2004.

SCHLUMPF, M. et al. In vitro and in vivo estrogenicity of UV screens. Environmental Health Persp. v. 109, p. 239-244, 2001.

SHARPE, R. M. et al. Abnormalities in functional development of the Sertoli cells in rats treated neonatally with diethylstilbestrol: a possible role for estrogens in Sertoli cell development. Biol. Reprod. v. 59, p. 1084-1094, 1998.

SHAW, G. M. Maternal workplace exposures to organic solvents and congenital cardiac anomalies. J. Occup. Med.Toxicol. v. 1, p. 371-376, 1992.
SKAKKEBAEK, N. E., RAJPERT-DE, M. E., MAIN, K. M. Testicular dysgenesis syndrome: an increasingly common developmental disorder with environmental aspects. Hum. Reprod. v. 16, p. 972-978, 2001.

SONNENSCHEIN, C., SOTO, A. M. An updated review of environmental estrogen and androgen mimics and antagonists. J. Steroid Biochem. Mol. Biol. v. 65, p.143-150, 1998.

SOTO, A. M., SONNENSCHEIN, C. Shining a light on sunscreens. Endocrinology. V. 146, p. 2127-2129, 2005.

SWAN, S. H. Intrauterine exposure to diethylstilbestrol: long-term effects in humans. A.P.M.I.S. v. 108, p. 793-804, 2000.

SWAN, S. H. et al. Decrease in anogenital distance among male infants with prenatal phthalate exposure. Environ. Health Persp. V. 113, p. 1056-1061, 2005.

VOM SAAL, F. S. et al. Implications for human health of the extensive bisphenol A literature showing adverse effects at low doses: a response to attempts to mislead the public. Toxicology V. 212, p. 244-252, 2005.

WIBBERLEY, D. G. et al. Lead levels in human placentae from normal and malformed births. Journal of Medical Genetics. V. 14, p. 339-345, 1977.

YAAKOV, B. et al. Major congenital malformations and residential proximity to a regional industrial park including a national toxic waste site: An ecological study. Environmental Health: A Global Access Science Source V. 5, p. 8-15, 2006.

Endereço para correspondência:
Sônia Corina Hess
DHT/CCET/UFMS
Caixa Postal 549
79070-900 Campo Grande - MS
- Brasil
Tel.: (67) 3345-7254
Fax: (67) 3345-7499
E-mail: schess@nin.ufms.br 